\title{
COLLABORATIVE HEALTHCARE PROCESS MODELLING: A CASE STUDY
}

José M. Framinan', Carlos Luís Parra ${ }^{2}$, Margarita Montes ${ }^{2}$, Paz Pérez ${ }^{2}$

${ }^{I}$ Industrial Management, School of Engineering - University of Seville <jose@esi.us.es> ${ }^{2}$ University Hospitals "Virgen del Rocío"- Seville redtelemedicina.sspa@juntadeandalucia.es, SPAIN

\begin{abstract}
Hospitals and health systems are trying to harmonise their information systems. However, most efforts have focused on data exchange, while little work has been carried out on the harmonisation of healthcare processes. Nonetheless, the latter may offer a number of advantages, such as the development of reference models containing best practices, and the fact that data exchange and systems integration can be more easily achieved on the basis of common (or similar) processes. In this communication, we present our experiences on collaborative modeling the current situation of healthcare process carried out within a national-wide network of Spanish hospitals. A methodology for collaboratively developing healthcare reference models is proposed, and the main findings in their application are presented.
\end{abstract}

\section{INTRODUCTION}

Many organizations consider electronic collaboration of distributed teams as a means to achieve higher productivity and improve the quality of their work products (Padgett, 2003). In this context, collaborative Business Process Redesign (BPR) appears to be a promising strategy for organisations to improve their products and services. BPR starts with a thorough understanding of the business processes of an organization (see e.g. Giaglis, 2001 for details). With this aim, the partners may share their expertise and develop a reference model that can be employed as benchmark for their Business Process and as a template for business process management. Healthcare environments have been involved in these changes too. Although hospital processes are complex and not very well known or categorised, the way to build a systemic view of hospital activities starts with the modeling of processes (Staccini et al, 2000). But individual process modelling is becoming insufficient. The need of enabling continuity of care among different hospitals and other healthcare providers has led hospitals and health systems to try harmonising their information systems. Establishing reference models for healthcare processes would make easier to manage and analyse current processes but it requires that a consensus among different workteams located in different hospitals or organizations must be reached. Our prime gain is to find an organizational ontology to interrelated healthcare processes and the characteristics they share. In addition to improve the way the services are actually delivered, for the healthcare sector there is another incentive for developing common reference models. Clearly, a processbased harmonisation may ease data exchange and systems integration, as data and functional requirements would be similar under a common process reference model. In this communication, we present our experiences on collaboratively developing reference models for healthcare processes. These experiences have been collected 
during the development of the FIS-G03/117 research project funded by the Spanish Ministry of Health. As part of the project, several healthcare organisations in Spain must develop common reference models for a number of processes, such as the care of certain chronic diseases. This collaboration among hospitals may be seen as a special type of Virtual Organization (VO), or even Virtual Enterprise (VE). Usually, in the VO/VE paradigm, a number of partners with different core competences collaborate to manufacture a product or deliver a service to what may be regarded as a common customer. In our case, the partners have common core competences (i.e. they all offer the same or similar services) while delivering their services to different customers (i.e. those citizens whose health is covered by the different hospitals).

\subsection{OBJECTIVES AND REQUIREMENTS}

The primary objective of our approach is to gain a global view of processes and to increase its comprehension, having people with different backgrounds and different approaches defining common processes with different viewpoints in different workplaces (Grützner et al, 2003). Specific objectives are to analyse patterns of healthcare activities, to build a systemic view of patient-centred processes, and to improve our knowledge and understanding of hospital organisation in order to achieve the effectiveness and efficiency of healthcare (Staccini et al, 2000).

Various requirements have been defined to successfully develop the virtual project:

1. The main requirement is to assist and advise the designers during their collective work. This is a key issue because the virtual process design we want to support is complex and structured, and most of process activities are creative and can be only modelled at a detailed level (Potts, 1989).

2. History-based assistance: all decisions, their rationale, and consequences are recorded as a basis for improving later decisions.

3. Flexibility and evolution: There are many decisions concerning how to structure the flow of tasks within a given context. Such structure must be incrementally constructed and dynamically evolvable. This is not only a technical issue but also an organizational question, particularly in a collective setting, for prescribing by whom, how, and why evolutions are undertaken (Longchamp and Denis, 1999).

Collaborative modelling focuses on the architecting and design of processes and systems using a formal modelling methodology. Three guidelines help to evaluate, compare and define collaborative system modelling solutions (Dori et al, 2004):

- Concurrency: The environment should allow team members to work on a shared system at the same time. The model should be available to all members in real-time, enabling them to get the most up-to-date view of the system.

- Communication: The environment should enable multi-way communication among the team members regardless of their physical whereabouts.

- Security: The environment should allow secure development, protecting the model under construction from unauthorized external entities and unauthorized changes by modellers.

\section{COLLABORATIVE PROCESS MODELING}

In Business Process Modeling, one should distinguish among methodology, 
technique, and tool (Kettinger et al, 1997). Modeling methodologies are supported by one or more techniques, and techniques can be supported by modelling tools (Giaglis, 2001). Despite the large number of vendors offering tools (see Kettinger et al., 1997), few -if any- of them offer some support for collaborative modelling (Walsh and Dickey, 2004). As it has been shown that employing a collaborative tool allows for wider user participation (Dean et al., 1997), we set this feature as a key one when selecting a tool for our project. Among the analysed tools, it turned out that only the IDS Scheer set of tools (ARIS Toolset ${ }^{\mathrm{TM}}$, and ARIS Web Designer ${ }^{\mathrm{TM}}$ ) supported, nonetheless only partially, the minimum requirements set for collaborative modelling. In ARIS, the modelling process itself can be achieved via Internet by employing a Java-based client (ARIS Web Designer ${ }^{\mathrm{TM}}$ ) that is able to interact with the ARIS Web Server and model a process through the Internet. Additionally, it can successfully integrate discrete-event simulation along with the static views of the models. The use of simulation for healthcare process modelling is often cited as a key issue, as then the models can capture the dynamic behaviour of the process (Ball et al, 2004). Finally, the software is probably the most extended tool in its class (see e.g. the survey by Gartner Group, 2001).

\section{A CASE STUDY FOR COLLABORATIVE HEALTHCARE PROCESS MODELING}

The healthcare quality paradigm requires not only the measurement of the outcomes of processes, but also the assessment of the means used to perform activities throughout each identified process. The needs for subsequent improvements in healthcare delivery, as for the reduction of variations in practices, stress the necessity for a continuous traceability of all care activities, and the detection, measurement and prevention of adverse events occurring during or after healthcare delivery (Staccini et al, 2000). The development of common reference models for healthcare processes is a challenging issue that differs from efforts in collaboratively modelling in other fields. Even if the main sketch of the process is similar to all hospitals, the large degree of freedom for organising resources in the hospitals has produced substantially different processes, particularly from the organizational and resource points of view. Other distinctive features are, among others: a high number of cooperating organizational units, limited resources, and a high ratio of manual activities (Amberg et al, 1996). Taking into account these specific features, healthcare process models can be assigned at various abstraction levels. In this project, we have considered three different levels:

- Meta-model level: a meta-model is an explicit model of the constructs and rules needed to build specific models within a domain of interest (Pidcock, 2003). It can be viewed as a set of building blocks and rules used to build models.

- Process Definition Model level. This level consists of a functional definition of healthcare processes built in each hospital or healthcare organization. Thereby, different points of view of the same process can be shared.

- Reference Model level. A reference model deals with a well-defined global representation of processes under consideration. Therefore, only one model, valid in any organization, will be used to describe a specific process. 


\subsection{STEPS FOR MODELLING}

\subsubsection{ORGANIZATION OF WORKING GROUPS}

Collaborative work among geographically-distributed teams has been widely studied in literature, which recognizes that while dispersed teams can bring benefits such as increased flexibility they also represent challenges such as difficulties with coordination (Ghosh et al, 2005). Successful teams can be described in terms of the behaviours performed by members, i.e. task-oriented behaviours, and maintenanceoriented behaviours (Townsend, 1999). In our project, virtual teams are organised in a structural way. Hence, we have been distinguished three types of working groups:

1. Design Working Groups (DWG). DWG are work teams devoted to building Process Definition Models. Thereby, each DWG has to model a process. Consequently, there is one DWG per partner (hospital) and process. For each DWG, there is a person in charge of the model (Harmon, 2003) that must accomplish tasks related to model publishing and decisions balloting. The process of model building that these WG should accomplish is described later on.

2. Coordination Working Group (CWG). Each CWG has to build and refine a reference model of a healthcare process. The CWG is composed by the Design Managers, who have been building the Process Definition Model of that process. The person responsible for the reference model is known as Coordination Manager, and the CWG members must consult him/her whenever weak points or potential improvements are discovered. Additionally, the CWG should develop a glossary of terms for the process, which contains all definitions of functions, event names and process information exchanged. We have selected SNOMED Clinical Terms $(B$ (Snomed, 2005) as common terminology, which is a validated clinical healthcare terminology and infrastructure that makes healthcare knowledge more usable and accessible. It provides a common language enabling a consistent way of capturing, sharing and aggregating health data across specialties and sites of care.

3. Integration Working Group. This group must revise and control different tasks being performed and build the first version of each reference model. The Integration Working Group supports maintenance-oriented behaviour, i.e. encouraging other group members; mediating differences among members; seeking to reach compromise with other members; encouraging contributions from reticent group members or limiting discussion on other subjects; evaluating the quality of group processes; or simply listening actively to discussion (Townsend, 1999)

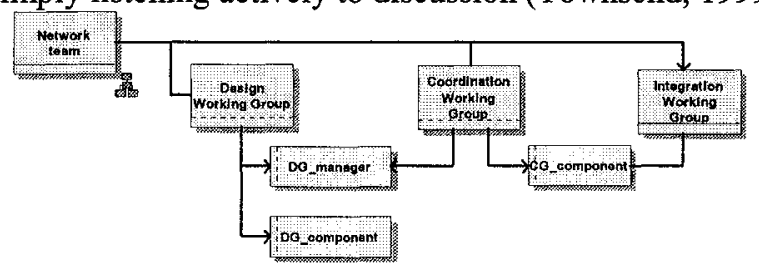

Figure 1 - Organizational Chart of virtual teams

We consider this WG to be a key issue for the involvement of all partners into the development of the reference models as it will help in developing a more representative model, which in turn will increase the chances of a successful implementation at a local level. The proposed structure for the virtual teams is 
shown in Figure 1 by means of an organisational chart.

\subsubsection{META-MODEL BUILDING}

The meta-model (see Figure 2) is used to describe entities that should represent the system elements: this is a key issue because each simulation tool has its own finite types of entities available to the modeller to be used in order to represent the real process components. It must include concepts for describing the aforementioned perspectives. The meta-model must rely on some consistent approach of design, and some homogeneity must be maintained without loosing creativity. A continuous feedback is required to keep the meta-model updated.

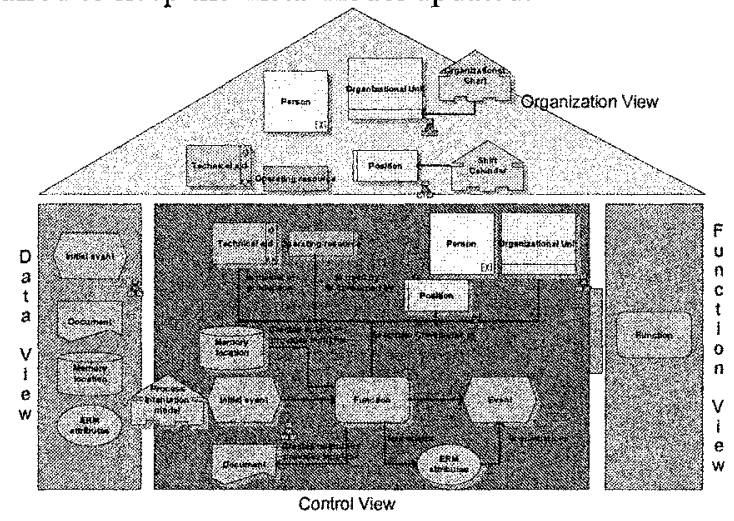

Figure 2 - Metamodel

\subsubsection{MODELLERS'TRAINING}

Training different modeller teams in the way they model their processes is a key issue in our collaborative modelling approach. Collaborative modelling requires enforcement of regulations that team members must follow in order to maintain the integrity of the process model under development (Townsend, 1999). Therefore, models cannot be built in an arbitrary way, but a commitment between freedom in modeling and the necessity of a guide followed by the project members to address the process modelling is required. For this reason, guidelines to build healthcare process modelling were developed within the context of the project, and two courses were held to explain them to all members.

\subsubsection{MODELS BUILDING}

According to the aforementioned guidelines, the following steps were accomplished: 1. Process selection: Each partner put forward the processes to be modeled, classified according to their importance, impact of their redesign on users, interest for continuous improvement efforts, etc. Then, after a consensus is reached, the processes to be modeled are assigned to different DWG, and the CWGs are created. 2. Modeling process objects description: In this step, stakeholders or organizational units whose value increases along the process, such as patients, relatives, professionals from another services or organizations, etc. are defined to facilitate reference model construction.

3. Setting the level of detail: The levels of detail required are established to each healthcare process. This level of detail depends mainly on the objectives of the 
study. It has to be noted that most of the differences between the same process performed in different hospitals does not lay on the sequence of the activities that are performed, but on how these activities are actually performed.

\subsubsection{REFERENCE MODELS BUILDING}

As mentioned before, the IWG builds the first version of the process reference models according to the different process definition models of the DWGs. These reference models are published both in the Network Web page, and in the Network Web Server, so it can be edited using ARIS Web Designer. Once these reference models are published, CWG members contribute to its improvement. After analysing the models, the CWG may agree on proposing changes to the model. Once the CWG considers the reference model to be representative of the real world, its validation is required. To do so, we suggest simulating the process. Every DWG involved in the project should simulate the established reference model in order to ensure that it fits into different healthcare organisations. Then, validation is approved and the definitive reference model version is published. If validation cannot be performed, a new iteration is needed. The procedure is shown in Figure 4.

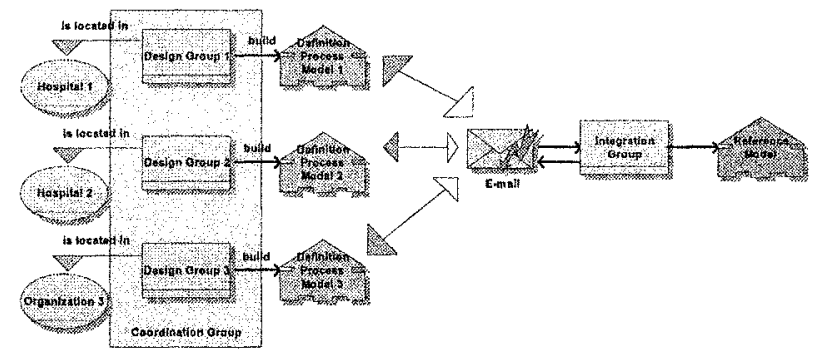

Figure 3 - Process Definition Process building scheme

\subsection{CHANGE MANAGEMENT}

An important requirement is the support of standard development processes, such as the spiral model. In such iterative development processes, each step in the process is based on refinement and modification of the output of the last step (Potok, 2002). According to this, the selected tool supports collaborative working by allowing models updates. Figure 4 shows a process model (described as an extended EventDriven Process Chain) on how these tasks have been accomplished. In this figure it can be seen how proposals are collected, then subsequently discussed, and, if approved, finally implemented.

\subsubsection{ACCESS CONTROL}

Access control is the process of limiting access to the resources of a system only to authorized users, programs, or processes, and therefore preventing activities that might lead to a breach of the system's security. Access control assumes that authentication of users has been verified. Authentication services are used to correctly determine the identity of a user (Cera et al, 2004). Collaborative modelling requires protecting the model from inspection and changes by unauthorized entities. In a collaborative environment, it is required that only authorized team members who are responsible for a specific development stage will be able to refine artefacts created at that stage (Dori et al, 2004). Access control policies commonly found can 
be classified as Discretionary Access Control (DAC) (Sandhu et al, 1996), Mandatory Access Control (MAC), and Role-based Access Control (RBAC) (Osborn et al, 2000). Based on DAC and RBAC policies, three profiles for controlling accesses have been defined in our project (see Table 2).

Table 2. Profiles assigned to groups

\begin{tabular}{|c|c|c|c|}
\hline Group & Access mode & Object & Task \\
\hline \multirow{2}{*}{ DWG } & Level 3 & $\begin{array}{c}\text { Their own Definition } \\
\text { Process Model }\end{array}$ & Manage their own models \\
\cline { 2 - 5 } & Level 1 & Reference models & Suggest improvement by mail \\
\hline \multirow{3}{*}{ CWG } & Level 2 & Reference models & Suggest improvement \\
\cline { 2 - 5 } & Level 1 & $\begin{array}{c}\text { Definition Process Model } \\
\text { from another CWG }\end{array}$ & Suggest improvement by mail \\
\hline \multirow{2}{*}{ IWG } & Level 3 & Reference models & Manage reference models \\
\cline { 2 - 5 } & Level 1 & Definition Process Model & $\begin{array}{c}\text { Refine semantic mistakes by } \\
\text { mail }\end{array}$ \\
\hline
\end{tabular}

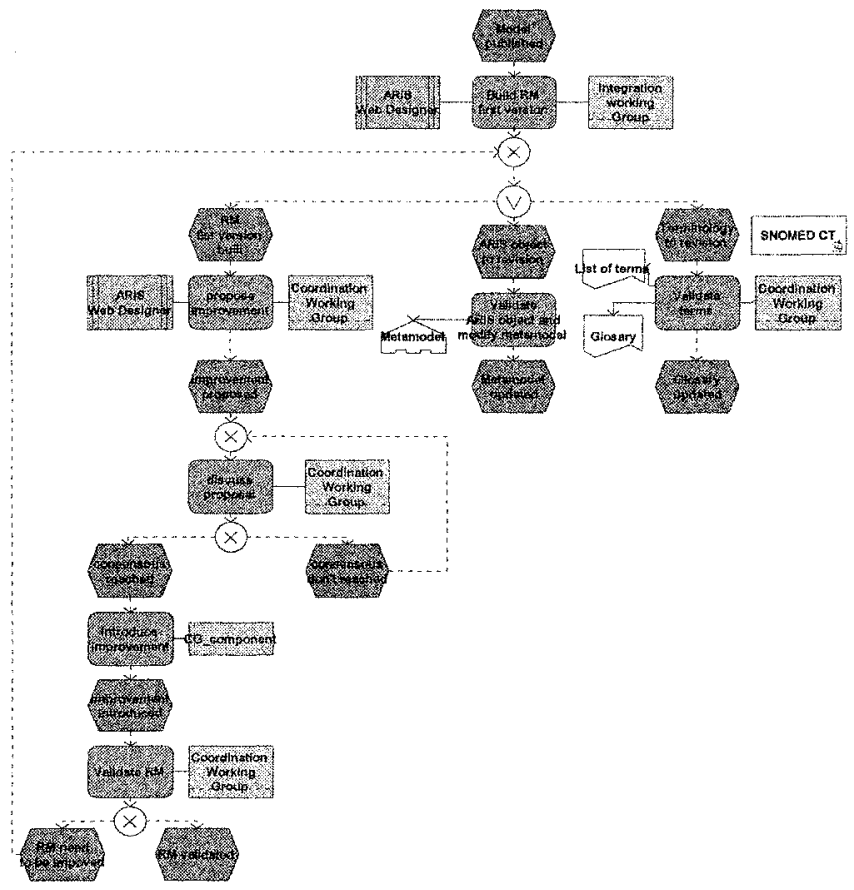

Figure 4 - Reference model

\section{FINAL REMARKS}

In this communication, we present an approach for collaboratively modelling healthcare processes. The approach stresses the need of participation of all partners so the model is representative, and a proper 'best practice' is really constructed. A number of mechanisms for carrying out the different steps in the approach, and the change management have been presented. Some ongoing work within this project 
includes developing common measures for assessing the impact that the implementation of the reference models may have on the hospital, and mechanisms for tracing the improvement after the implementation.

In this case study, the implementation of a virtual team has led to improve work developing, to decrease costs (for example, reducing travel time), to short life cycles (transforming from series processes to parallel processes), to encourage innovation (allowing the most diverse participation in the project and stimulating creativity) and to focus learning (capturing knowledge through natural course of working, developing or improving accesses to experts).

\section{REFERENCES}

1. Amberg, Michael and Gräber, Stefan. Specifying Hospital Information Systems Using Business Process Modeling. Brender, J., Christensen, J. P., Scherrer, J. R., and McNair, P. 1037-1041. 1996. Amsterdam, IOS Press. Medical Informatics Europe 96.

2. Ball, Peter, Albores, Pavel, and Macbryde, Jill. Requirements for modelling e-Business processes. Production Planning \& Control 15[8], 776-785. 2004.

3. Cera, Christopher D., Kim, Taeseong, Han, JungHyun, and Regli, William C. Role-based viewing envelopes for information protection in collaborative modeling. Computer-Aided Design 36[9], 873-886. 2004

4. Dori, Dov, Beimel, Dizza, and Toch, Eran. OPCATeam - Collaborative Business Process Modeling with OPM. 66-81. 15-7-2004. BPM 2004, Springer-Verlag.

5. Ghosh, Tanu, Yates, JoAnne, and Orlikowski, Wanda. Using communication norms for coordination: Evidence from a distributed team. Electronic Citation. http://seeit.mil.edu

6. Giaglis, GM. A taxonomy of business process modeling and information systems modeling techniques. International Journal of Flexible Manufacturing Systems 13[2], 209-228. 2001. Elsevier Science B.V.

7. Grützner, Ines, Münch, Jürgen, Fernandez, Alejandro, and Garzaldeen, Badie. Guided Support for Collaborative Modeling, Enactment and Simulation of Software Development Processes. 3-52003. Prosim 2003, Workshop. 2003.

8. Harmon, Paul. Business Process Change: A Manager's Guide to Improving, Redesigning and Automating Processes. Morgan Kauffman

9. Kettinger, William J., Teng, James T. C., and Guha, S. Business Process Change: a study of methodologies, techniques, and tools. MIS Quarterly 21[1], 55-80. 1997.

10. Osborn, S. L., Sandhu, R. S., and Munawer, Q. Configuring role-based access control to enforce mandatory and discretionary access control policies. Information System Security 3[2], 85-106. 2000.

11. Padgett, Gerson David. Informe resumen de tecnología de información para negocios Tecnología: Groupware. Electronic Citation. http:/www.monografias.com

12. Pidcock, Woody. What are the differences between a vocabulary, a taxonomy, a thesaurus, an ontology, and a meta-model? Electronic Citation. http:/www.metamodel.com

13. Potok, T. E. Extensions to the spiral model to support joint development of complex software systems. 2002. Proc. of the 30th Annual Southeast Regional Conference, ACM Press 2002.

14. Potts, C. A Generic Model for Representing Design Methods. Proceedings of 11 th ICSE.

15. Sandhu, R. S., Coyne, E. J., Feinstein, H. L., and Youman, C. E. Role-based access control models. IEEE Comput 29[2], 38-47. 1996.

16. Snomed. SNOMED. Electronic Citation. SNOMED® International 24-5-0005. hitp://www.snomed.org/.

17. Staccini, Pascal, Joubert, Michel, Quaranta, Jean-François, Fieschi, Dominique, and Fieschi, Marius. Integration of Health Care Process Analysis in the Design of a Clinical Information System: Applying to the Blood Transfusion Process. Proc. AMIA Symposium 2000.

18. Townsend, Christine. Teams in Agricultural Education: An Assessment of Team Process Instruction. Proc. 26 Annual National Agricultural Education Research Conference. 1999. 